\title{
BMJ Open Anaesthetic protocol for paediatric glaucoma examinations: the prospective EyeBIS Study protocol
}

\author{
Nina Pirlich, ${ }^{1}$ Franz Grehn, ${ }^{2}$ Katja Mohnke, ${ }^{1}$ Konrad Maucher, ${ }^{1}$ Alexander Schuster, ${ }^{2}$ \\ Eva Wittenmeier, ${ }^{1}$ Irene Schmidtmann, ${ }^{3}$ Esther M Hoffmann (1) ${ }^{2}$
}

To cite: Pirlich N, Grehn F, Mohnke K, et al. Anaesthetic protocol for paediatric glaucoma examinations: the prospective EyeBIS Study protocol. BMJ Open 2021;11:e045906. doi:10.1136/ bmjopen-2020-045906

- Prepublication history for this paper is available online. To view these files, please visit the journal online (http://dx.doi org/10.1136/bmjopen-2020045906).

Received 15 0ctober 2020 Accepted 04 September 2021

Check for updates

(c) Author(s) (or their employer(s)) 2021. Re-use permitted under CC BY-NC. No commercial re-use. See rights and permissions. Published by BMJ.

${ }^{1}$ Department of Anaesthesiology, University Medical Centre of the Johannes Gutenberg University Mainz, Mainz, Germany ${ }^{2}$ Department of Ophthalmology, University Medical Centre of the Johannes Gutenberg University Mainz, Mainz, Germany ${ }^{3}$ Institute of Medical Biostatistics, Epidemiology and Informatics, University Medical Centre of the Johannes Gutenberg University Mainz, Mainz, Germany

Correspondence to Dr Esther M Hoffmann; ehoffman@uni-mainz.de

\section{ABSTRACT}

Introduction Neonates and young infants with diagnosed or highly suspected glaucoma require an examination under anaesthesia to achieve accurate intraocular pressure (IOP) measurements, since crying or squinting of the eyes may increase IOP and lead to falsely high values. IOP considerably depends on perioperative variables such as haemodynamic factors, anaesthetics, depth of anaesthesia and airway management. The aim of this paper is to report the design and baseline characteristics of EyeBIS, which is a study to develop a standardised anaesthetic protocol for the measurement of IOP under anaesthesia in childhood glaucoma, by investigating the link between the magnitude of IOP and depth of anaesthesia.

Methods and analysis This is a single-centre, prospective cohort study in 100 children with diagnosed or highly suspected glaucoma all undergoing ophthalmological examination under general anaesthesia. 20 children, who undergo general anaesthesia for other reasons, are included as controls. The primary outcome measure is the establishment of a standardised anaesthetic protocol for IOP measurement in childhood glaucoma by assessing the relationship between IOP and depth of anaesthesia (calculated as an electroencephalography variable, the bispectral index), with special emphasis on airway management and haemodynamic parameters. The dependence of IOP under anaesthesia on airway management and haemodynamic parameters will be described, using a mixed linear model. Restricting the model to patients with healthy eyes will allow to determine a $95 \%$ reference region, in which $95 \%$ of the measurement values of patients with healthy eyes can be expected.

Ethics and dissemination The study has been approved by the local ethics committee of the Medical Association of Rhineland-Palatine (Ethik-Kommisssion der Landesaerztekammer Rheinland-Pfalz), Germany (approval number: 2019-14207). This work will be disseminated by publication of peer-reviewed manuscripts, presentation in abstract form at national and international scientific meetings and data sharing with other investigators.

Trial registration number ClinicalTrials.gov Registry (NCT03972852).

\section{INTRODUCTION}

Background and rationale

A basic requirement for diagnosis, monitoring and therapy of childhood glaucoma is the accurate measurement of intraocular pressure

\section{Strengths and limitations of this study}

- EyeBIS will be the first study investigating the measurement of intraocular pressure (IOP) in neonates and young infants taking into account the complexity of multifactorial disruptive perioperative factors.

- EyeBIS will provide data on distribution of paediatric IOP.

- The developed protocol may provide a recommendation for other glaucoma centres.

- It is only a single-centre observational study.

(IOP). Despite newer less invasive measurement techniques (rebound technology), neonates and young infants still require an examination under anaesthesia (EUA), either under sedation or general anaesthesia.

Squinting and the elevated stress level following the release of catecholamines may lead to falsely high IOP values and subsequently to inadequate therapy. Success rates for correct measurement of IOP in awake children vary between $14 \%$ and $60 \%$ in the literature. ${ }^{12}$ As congenital glaucoma damage in newborns and young children is exclusively IOP related (ie, Descemet tears, optic nerve head damage), accurate measurement of IOP under EAU is crucial.

To date, there are no prospective studies from which detailed recommendations on standardised general anaesthesia in children with glaucoma may be derived.

Perioperative anaesthetics include all inhalation anaesthetics and most centrally depressing drugs such as propofol, benzodiazepines and opioids. They result in a reduction of IOP in both healthy and glaucomatous eyes. ${ }^{3}$ The depth of anaesthesia and IOP reduction are correlated. IOP-lowering effects depend on the applied dose and on the time of administration. ${ }^{4-7}$ The effects of propofol on IOP are mainly known in adult patients, where IOP reduction is suspected to be associated with the lowering of the mean arterial pressure. ${ }^{8}$ Available data on the effect of 
propofol on IOP in healthy children are inconsistent and there are no data in children with glaucoma. ${ }^{9}$ Furthermore, there are no data on the influence of opioids on the IOP of children with glaucoma.

When applying anaesthesia, airway management also has an influence on IOP. ${ }^{3}$ Laryngoscopy and intubation can increase IOP substantially, especially when it comes to coughing. However, IOP may rise even without an externally detectable reaction such as a sympathicotonic cardiovascular mechanism, especially with shallow anaesthesia. ${ }^{10-12}$ Laryngeal masks are widely used in adult and paediatric respiratory management and considered safe by paediatric anaesthesiologists in a variety of clinical settings. ${ }^{13}$ Compared with endotracheal intubation in children, the use of laryngeal masks is associated with less cardiovascular reactions and a lesser increase in IOP. ${ }^{14}$

The EyeBIS Study will be the first study to employ a strict standardised protocol for anaesthesia in children with glaucoma. The study will assess the relationship between depth of anaesthesia and IOP in this population. To determine depth of anaesthesia, the bispectral index (BIS) is used. The index has a range of $0-100$ and is a calculated electroencephalography (EEG) variable, used to measure the effects of anaesthetics. A BIS score below 60 indicates the degree of hypnosis of EUA. Both Schäfer et al and Hanna et al have described the need to investigate the relationship between depth of anaesthesia and IOP. ${ }^{615}$ A correlation between IOP and depth of anaesthesia using BIS in children is already available. ${ }^{16}$ The purpose of this manuscript is to report on this protocol in the population described below.

\section{METHODS AND ANALYSIS}

\section{Study setting and design}

The Department of Ophthalmology, University Medical Center Mainz, has developed an expertise in childhood glaucoma diseases. In the years 2016 and 2017, surgery was performed in approximately 80 children. Since the founding of the German Childhood Glaucoma Centre at the University Medical Center Mainz in June 2017, the number of children receiving a glaucoma diagnosis or surgery has increased significantly. The collaboration between paediatric ophthalmologists and paediatric anaesthesiologists has led to an enhanced focus and expertise in this area and identified the necessity to develop a standardised protocol for general anaesthesia while performing IOP measurements. Established and safe anaesthesia regimens have been modified, taking into account various known factors that influence the measurement of IOP.

With the planned study, we would like to evaluate whether reliable and reproducible measurement values can be generated by a standardised protocol. The EyeBIS trial is a prospective, single-centre, non-randomised clinical trial.

\section{Patient and public involvement}

Patients of this research were first involved in June 2019 by the research team (NP and EMH, and medical students). Eligible patients (see section below) were asked whether they are interested in this study. Inclusion criteria were discussed with parents and children and patients were examined according to the individual standards and standard operating procedures (SOPs) of the Clinic for Anaesthesiology and the Department of Ophthalmology. Patients were not involved in the development and design of the study protocol. The public was not involved. Study results will not be disseminated to participants specifically. However, if participants are interested in the results of the research, they will receive any information, the manuscript and published research on this topic in the future.

\section{Trial population and eligibility criteria}

Children in the age group 6 months-10 years will be included, when meeting the following criteria: requirement of EUA with a laryngeal mask for a surgical or diagnostic procedure; suspected glaucoma or control children undergoing ocular surgery other than glaucoma surgery (control group); American Society of Anesthesiologists classification 1, 2 or 3; and informed written consent from one of their legal representatives.

Patients will not be included in this trial if they meet one or more of the following criteria: contraindications to the use of a laryngeal mask (eg, severe infections; tumour or bleeding in the upper airway tract, which might prevent the sufficient placement of the laryngeal mask; if the expected magnitude of ventilation pressure exceeds the upper leakage pressure of the laryngeal mask $\left(40 \mathrm{~cm} \mathrm{H}_{2} \mathrm{O}\right)$; the necessity of a constant tracheal access; patients with a severe gastro-oesophageal reflux; or interference of the laryngeal mask with the surgical approach), or known allergy to propofol or remifentanil. Eligibility criteria are shown in figure 1 .

\section{Recruitment and participant timelines}

Participant inclusion started in June 2019 in the Childhood Glaucoma Centre at the University Medical Center, Mainz, Germany. The history and physical examinations of all patients scheduled for surgery were screened preoperatively for predictors of difficult airway, oesophageal reflux and allergies.

Patient will be informed about the study by the investigators and medical students (EMH, NP). Patients will be included if they require the insertion of a laryngeal mask under general anaesthesia. Informed consent for all paediatric participants will be obtained from one of their legal representatives. This also includes information and consent according to the German Medical Privacy Rules (in analogy to the US Insurance Portability and Accountability Act of 1996). Prospective approval of the study will be granted by the local ethics committee of the Medical Association of the Rhineland-Palatine state (Ethik-Kommission der Landesaerztekammer Rheinland-Pfalz), Germany (approval number: 2019-14207).

The schedule of study enrolment is shown in figure 2.

\section{Intervention}

Induction and maintenance of EUA is performed. In the present study, the laryngeal mask Ambu AuraGain 


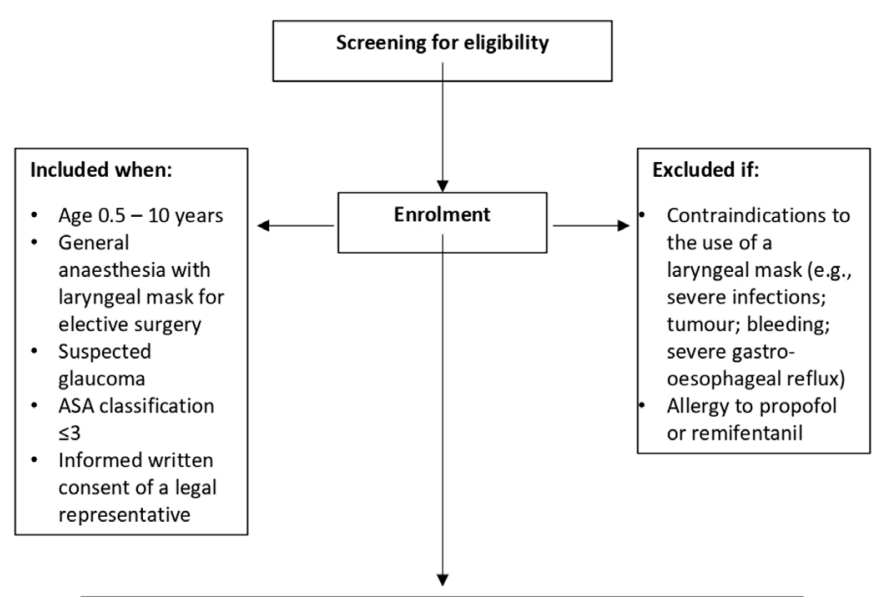

Primary outcome measure
- The primary outcome measure is the association between
intraocular pressure and the bispectral index.
Secondary outcome measures
- Comparison of two different examination tools (applanation
tonometry and rebound tonometry)
Magnitude of the paediatric intraocular pressure during general
anaesthesia regarding
- Cofining the normal range of the paediatric intraocular pressure
rather regression of the intraocular pressure on the corneal
thickness

Figure 1 Study flow chart according to the American Society of Anesthesiologists (ASA) with inclusion and exclusion criteria, as well as outcome measures.

(German distribution by Ambu, Bad Nauheim, Germany) is used, which is a second-generation laryngeal mask with integrated gastric access and the possibility of fibreoptic intubation. ${ }^{17}$ Compression of the cervical vessels through the cuff of laryngeal masks has been reported in adults. ${ }^{18}$ It has not been investigated whether the blockage of a laryngeal mask in children has an influence on IOP by obstructing the venous return. According to the SOPs of the Department of Anaesthesiology, a premedication with oral midazolam (juice) is administered when the patient is collected from the ward. A dosage of $0.5 \mathrm{mg} / \mathrm{kg}$ (up to a maximum dose of $10 \mathrm{mg}$ ) is given.

Local anaesthetics are applied topically to two possible puncture sites at least 1 hour prior to surgery. Before induction of anaesthesia, intravenous access is established. The child's head is positioned in a neutral way in a head ring, with the body in a flat back position. To optimise mask ventilation, the shoulder blades are padded with a rolled-up surgical tissue.

The induction and maintenance of EUA is performed by an anaesthesiologist, specifically educated in anaesthesia for neonates and young infants. Before initiating the EUA, pulse oximetry monitoring, ECG, non-invasive blood pressure measurement and the BIS are started.

Preoxygenation takes place with a fractional inspired oxygen $\left(\mathrm{FiO}_{2}\right)$ of 0.8 and a fresh gas flow of $7 \mathrm{~L} / \mathrm{min}$.

As soon as the BIS measurement starts, the BIS value and an initial blood pressure value are noted.

If it is possible to establish intravenous access, an initial propofol bolus of $2-4 \mathrm{mg} / \mathrm{kg}$ body weight is applied. The

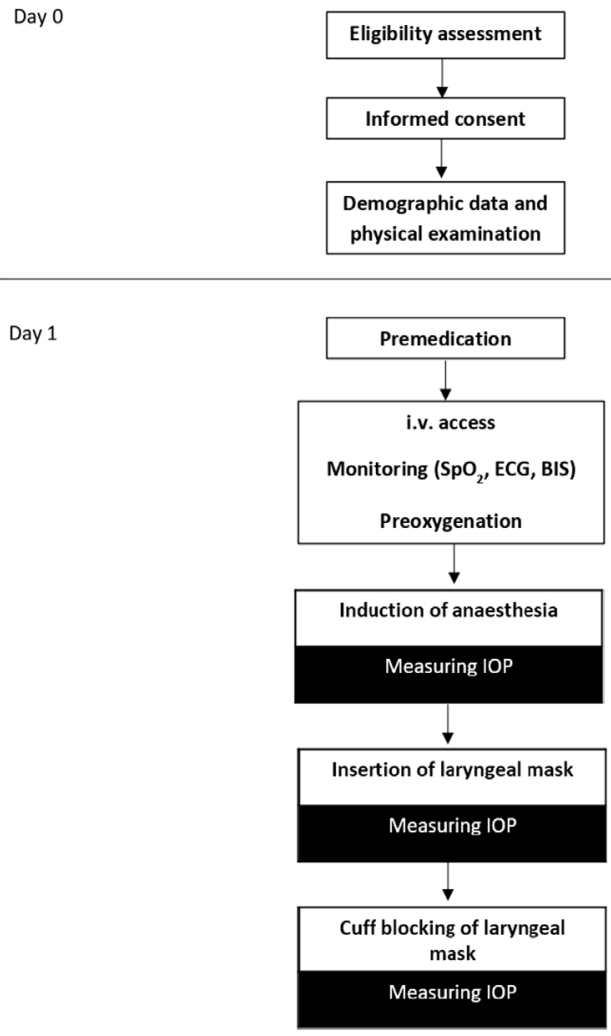

Figure 2 Schedule of study enrolment and interventions. $\mathrm{BIS}$, bispectral index; IOP, intraocular pressure; i.v., intravenous; $\mathrm{SpO}_{2}$, oxygen saturation.

children are breathing spontaneously. When the oxygen saturation drops below $90 \%$, a ventilation via a face mask takes place.

The schedule of interventions is also summarised in figure 2.

IOP measurements take place during different depths of anaesthesia. Measurement is taken by one experienced examiner per child (KM, AS, EMH). A first IOP measurement is performed when sufficient sedation for IOP measurement after titrated propofol application (2-4 mg/ $\mathrm{kg}$ or more if necessary) is achieved. A second IOP measurement is carried out $60 \mathrm{~s}$ after placement of the laryngeal mask to avoid a potentially falsely high IOP value due to a direct sympathicotonic reaction caused by the laryngeal mask placement. Then, a third IOP measurement is taken after a break of $60 \mathrm{~s}$ directly after blockage of the cuff of the laryngeal mask $\left(60 \mathrm{~cm} \mathrm{H}_{2} \mathrm{O}\right)$.

IOP measurement of each eye is performed with the iCare PRO Tonometer followed by a measurement with the Perkins applanation tonometer after a 1 min waiting time to avoid potential influence of repetitive measurements on IOP.

Central corneal thickness (CCT) has an influence on IOP measurement in adults and children and is measured in this study. ${ }^{19}$ CCT has also been shown to be a relevant factor in the evaluation of childhood glaucoma in many studies and appears to result in significant differences in the measurement of IOP, depending on the device used. ${ }^{20}$ This is why two different devices are used in this study. All 
IOP measurements are performed by one of three expert study investigators.

The Perkins Mk3 is available for measuring IOP. The Perkins Mk3 is a mobile, battery-powered applanation tonometer, which consists of the following components: a forehead support for correct placement, a handgrip for the examiner, a LED (light-emitting diode) light source with a blue filter, a biprism and a force transducer. The force transducer measures how much force the examiner must use to flatten the cornea to a defined circular area of $4.8 \mathrm{~mm}^{2}$ (diameter $3.06 \mathrm{~mm}$ ). For the visualisation of the edge of this surface, fluoresceine (a fluorescent dye) is applied in advance to the cornea. In compliant adults, the awake patient is examined after topical application of a local anaesthetic.

The system draws on the 1965 prototype developed by Perkins. ${ }^{21}$ It has been specifically designed to measure IOP in patients who cannot adequately sit upright at a standard stationary applanation tonometer.

The iCare PRO Tonometer (iCare Finland Oy, 01510 Vantaa, Finland, German distribution by bon Optic Vertriebsgesellschaft, Lübeck, Germany) has been available for inpatient and outpatient as well as self-tonometry since its certification in 2010. The iCare PRO Tonometer is a mobile, battery-operated rebound or induction tonometer, which consists of the following components: a forehead support for correct placement, a handle for the examiner and a miniaturised measuring head. The measuring head bounces against the cornea from a short distance in six, very short individual measurements. Depending on the IOP, the measuring head is slowed down to varying degrees, from which the device calculates the IOP using magnetic coils. The compliant awake patient does not require local anaesthesia. Only a few studies have compared the two techniques in children. ${ }^{22} 23$

The agreement between the instruments (Perkins applanation tonometry and iCare rebound tonometry) has been evaluated only in a few studies under different conditions from our study. Rebound tonometry has been shown to overestimate IOP in high IOP values.

\section{CCT measurement}

Measurement of CCT is performed by Tomey AL-3000 (Tomey, Nuremberg, Germany). The SP-3000 is an ophthalmic diagnosis instrument which acquires corneal thickness, by using ultrasonic waves that are transmitted from the ultrasonic oscillator enclosed in the probe. One measurement is performed in each eye.

In addition to IOP measurements, BIS values, blood pressure (including mean arterial pressure), heart rate and oxygen saturation are documented for each time point of IOP measurement. General anaesthesia is maintained with a syringe pump of propofol at a rate of $4-5 \mathrm{mg} / \mathrm{kg} / \mathrm{hour}$ and a syringe pump of remifentanil with a running rate of $0.3 \mu \mathrm{g} / \mathrm{kg} / \mathrm{min}$. In case an intravenous access is initially not possible, mask induction of EUA via sevoflurane ( 4 vol $\%$, flow $7 \mathrm{~L} / \mathrm{min}, \mathrm{FiO}_{2} 0.8$ ) is performed, followed by the establishment of an intravenous access.

Figure 3 presents all interventions on a time scale.

\section{BIS monitoring}

The study uses the Aspect XP Bispectral Index Monitor (Medtronic, Minneapolis, Minnesota, USA). It is available for intraclinical brain function monitoring by deriving raw EEG signals from patients for perioperative or intensive care monitoring, as well as for clinical research.

As described in the introduction, the BIS, with a dimension of $0-100$, is a calculated EEG variable and can be used to aid in assessing the effects of anaesthetics. A BIS score below 60 indicates the degree of hypnosis of general anaesthesia. The measurement is carried out by a sensor fitted for the paediatric anatomy (BISTM Paediatric Sensor), which is mounted on the forehead of the patient.

Data from children who had to undergo mask induction are excluded from the main analysis and analysed separately. Criteria to cancel the intervention include an unexpectedly difficult airway, an unrecoverable laryngeal mask leak, a different need for endotracheal intubation and circulatory instability requiring intervention.

\section{Outcome measures}

Primary outcome of the study is the correlation between BIS and IOP under standardised anaesthetic conditions.

Aim of this protocol paper is to report design and baseline characteristics of EyeBIS, a study to develop a standardised anaesthetic protocol for the measurement of IOP under general anaesthesia in childhood glaucoma by investigating the partial correlation between the magnitude of IOP and depth of anaesthesia adjusting for potential confounders.

Secondary outcome measures include the comparison of two different IOP measurements methods (applanation tonometry and rebound tonometry), the magnitude of the paediatric IOP during EUA in relation to cuff pressure of the laryngeal mask, regarding end expiratory $\mathrm{CO}_{2}$ pressure, blood pressure and heart frequency, effect size of midazolam premedication and the definition of the normal range of the paediatric IOP, as well as the correlation of the CCT and IOP (determined by regression of IOP on CCT).

Primary and secondary outcomes are also shown in figure 1.

\section{Data collection, management and analysis}

\section{Data collection and management of the present study}

For data collection, a paper-based case report form (CRF) was developed and is used for each patient. In addition to all other documentation, the CRF and the patient's study file belongs to the source data. The CRF contains information that is requested directly from the patient and thus cannot be verified on the basis of the patient's study file (screening information and data collected in the operating room). 


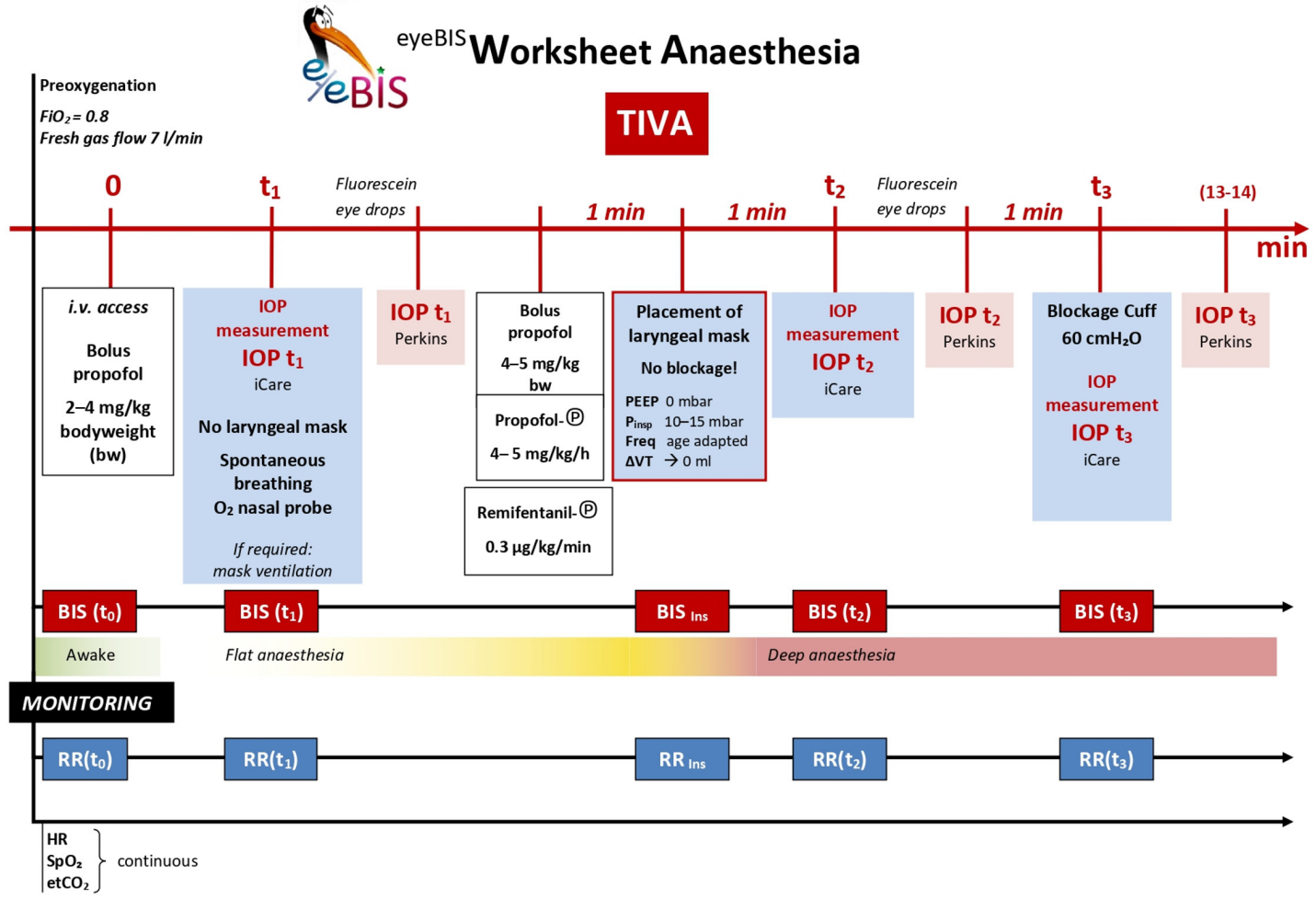

Figure 3 EyeBIS worksheet. Detailed layout of all steps on the interventional time scale. BIS, bispectral index; etCO ${ }_{2}$, end tidal carbon dioxide; $\mathrm{FiO}_{2}$, fractional inspired oxygen; $\mathrm{HR}$, heart rate; IOP, intraocular pressure; i.v., intravenous; PEEP, Positive End-Expiratory Pressure; $\mathrm{P}_{\text {insp }}$, Inspiratory pressure; $\mathrm{RR}$, blood pressure; $\mathrm{SpO}_{2}$. oxygen saturation; TIVA, total intravenous anaesthesia; VT, tidal volume.

For pseudonymisation purposes, every patient is coded with a specific patient number. In addition to the paperbased form, this study is also documented electronically. For this purpose, all information from the study file and the CRF is transferred to the computer in a tabular form.

\section{Access to data}

Data management of the present study is performed by the main investigator. All electronically stored data are backed up regularly. The pseudonymity of patients is ensured throughout the evaluation. All study data, including the electronically recorded data, will be archived and kept for at least 15 years after completion of the study according to the currently valid International Council for Harmonisation (ICH) Guidelines on Good Clinical Practice (GCP) E6 (R2). Data are accessible to all participating personnel and monitors. The database management system is capable of producing accurate and complete copies of the data in visual form for inspection by government agencies or ethics committees. Enrolled patients and their authorised representative have been informed about this.

\section{Monitoring}

Prior to enrolling patients, the investigators were briefed on the CRF and study protocol. All documents required for data collection are available in the operating theatre. Each CRF is filled in by the investigator after the measurements have been performed. The data are then promptly entered electronically under his supervision.
The investigator regularly evaluates the progress of data collection and study outcomes in order to address any emerging data collection issues at an early stage. The data monitoring is managed and analysed in accordance with the ICH GCP Guideline E6 (R2) and followed the requirements of German Drug Law.

Adverse events will be recorded after patient enrolment. The study will be temporarily interrupted by the attending investigator at any time on the individual subject, if a serious adverse event is suspected, which may be associated with IOP measurement or an airway device being used. A suspected adverse event or adverse reaction will be considered serious when it comes to one of the following events: death, a life-threating reaction leading to inpatient hospitalisation, and a persistent or significant incapacity or substantial disability of the normal ageadapted life functions. If the protocol is discontinued as a result of an adverse event, study personnel will document the circumstances and data leading to the discontinuation of measurement. The principal investigator will inform the local research ethics committee in case of a severe adverse event following local SOPs.

The Clinical Research Unit of the Department of Anaesthesiology, University Center Mainz inspects and reviews screening forms and clinical data at regular intervals.

\section{Sample size considerations}

With the envisaged number of 100 subjects, a single IOP measurement of one eye and parallel determination of the 
BIS, the null hypothesis 'correlation $=0$ ' can be rejected in a two-sided test at the $5 \%$ level with $86.5 \%$ power if the correlation amounts to 0.3 . If the actual correlation is 0.35 , the power rises to $95 \%$. The multiple measurements provide additional information, resulting in a power gain. It is not yet possible to anticipate how strong the correlation between the multiple measurements will be. With decreasing correlation between repeat measurements power increases and, similarly, also smaller correlation between IOP and BIS will be detectable for fixed powerhow small depends on the yet unknown correlations. For the control group, we selected 20 children, having feasibility in mind. We will, however, include further children in this group.

For statistical analysis, SAS V9.4 statistical software will be used. The analysis corresponds to Strengthening the Reporting of Observational Studies in Epidemiology statement for observational studies.

\section{Description of patient group at baseline}

The baseline features of patients will be described using absolute numbers (n) and percentages for categorical variables and the minimum, maximum, mean and $\mathrm{SD}$ for normally distributed variable and as median (IQR) for non-Gaussian variables. The Pearson correlation coefficient will be used to compare patient specifics between the groups and the baseline.

\section{Analysis of the primary outcome}

The relationship between the IOP and BIS will be investigated using a mixed linear model including measurements at all three time points. In this model, IOP is the dependent variable, the BIS is the main independent variable. Random effects are subject and eye (of a subject). Time per se is not of interest as essentially the depth of anaesthesia measured by BIS at each time point is of interest. Time will be considered by taking repeated measurements into account. Further, we will adjust for age (quantitative), sex, CCT and cumulative midazolam dose administered until the time of measurement. Eyes within a patient are likely not to be independent, therefore a random patient effect is included in the model.

\section{Analysis of the secondary outcome}

To compare the two measurement methods for IOP, applanation tonometry and rebound tonometry, BlandAltman diagrams will be created.

The dependence of IOP under general anaesthesia on the cuff pressure of the laryngeal mask, the end tidal $\mathrm{CO}_{2}$ partial pressure, the blood pressure and the heart rate will also be described by a mixed linear model, with the variables mentioned above as covariates and random effects for subject and eye and adjustment for corneal thickness. The general considerations for the primary outcome apply here, too.

For children without glaucoma, a quantile regression will be performed that takes into account the factors mentioned above. From this, it is possible to deduce standard ranges in which, for example, $90 \%$ or $95 \%$ of the values of healthy children are expected.

\section{Subgroup analysis}

Data from children who had to undergo mask induction will be analysed separately.

\section{DISCUSSION}

Several studies have documented various variables that have an impact on the paediatric IOP. ${ }^{3}$ The weakness of the previously published studies is that none has examined all in a single study setting. To our current knowledge, the EyeBIS Study is the only clinical study of its kind to associate IOP in 100 patients with childhood glaucoma and 20 patients without glaucoma (control group) with the depth of anaesthesia under the best possible standardised environmental conditions, due to the exclusivity and safety of our patient population.

In conclusion, if our study will find a partial correlation between BIS and IOP, this could lead to more reliable IOP data in childhood glaucoma EUA. This protocol could be a reference standard for children with suspected glaucoma who cannot undergo an examination while awake. This leads to an improved, more reliable ability to diagnose glaucoma with an earlier therapy, which overall leads to a significantly better functional outcome for children.

\section{ETHICS AND DISSEMINATION \\ Research ethics approval}

The requirements of the ICH Guideline for GCP E6 of June 1996 and of Committee for Proprietary Medicinal Products (CPMP)/ICH/135/95 of September 1997 are, in addition to the national laws and the Declaration of Helsinki (Sommerset West 1996), the basis for carrying out this study.

All study personnel are obliged to participate in this study according to these guidelines.

\section{Consent or assent}

Before being included in the study, the study will be verbally and comprehensibly explained to patient and one of his/her authorised representatives by a clinical study investigator, as required by German law. He/she will also receive a comprehensively written information sheet. The authorised representatives will have the opportunity to have an informed discussion with the clinical study investigator about the study.

The clinical study investigator will obtain written consent from the authorised representatives willing to participate in the trial. The information leaflet and a new execution of the consent document will be handed over to one of the authorised representatives. Upon request, the patient will receive a child-friendly version of the information leaflet. 
The authorised representative may withdraw from the study at any time if he/she is unwilling to continue in the trial. In this case, the data from a patient who requests full withdrawal will not be considered in the data analysis.

\section{Confidentiality}

All original documents will be kept in the clinical research unit for the next 15 years. The study data will be handled as requested by the German Federal Data Protection Act, which implements the Directive 95/46/EC on data protection (Data Protection Directive). All original records will be kept on file at the trial sites or coordinating data managing centre for 15 years. The cleaned electronic trial database file will be anonymised and kept on file for 15 years.

Acknowledgements The authors would like to thank all involved doctors and nurses of the Department of Anaesthesiology and Ophthalmology for their great effort and support for this study. They also thank Patricia Buchholz for writing support.

Contributors The study concept and design were conceived by NP, EMH, KaM, IS and EW. NP, EMH and KoM are conducting screening and data collection. Analysis and interpretation of data will be performed by IS, EW, NP, EMH, KaM, FG and AS. KaM and NP prepared the first draft of the manuscript. All (NP, FG, EMH, KaM, IS, EW, AS, KaM, KoM) authors have provided edits and critiqued the manuscript for intellectual content, as well as have given final approval for manuscript submission. Results of this study will be part of the doctoral thesis of KaM.

Funding The authors have not declared a specific grant for this research from any funding agency in the public, commercial or not-for-profit sectors.

Competing interests None declared.

Patient and public involvement Patients and/or the public were not involved in the design, or conduct, or reporting, or dissemination plans of this research.

Patient consent for publication Parental/guardian consent obtained.

Provenance and peer review Not commissioned; externally peer reviewed.

Open access This is an open access article distributed in accordance with the Creative Commons Attribution Non Commercial (CC BY-NC 4.0) license, which permits others to distribute, remix, adapt, build upon this work non-commercially, and license their derivative works on different terms, provided the original work is properly cited, appropriate credit is given, any changes made indicated, and the use is non-commercial. See: http://creativecommons.org/licenses/by-nc/4.0/.

ORCID iD

Esther M Hoffmann http://orcid.org/0000-0002-0949-4268

\section{REFERENCES}

1 Sihota R, Tuli D, Dada T, et al. Distribution and determinants of intraocular pressure in a normal pediatric population. $J$ Pediatr Ophthalmol Strabismus 2006;43:14-18.
2 Oberacher-Velten I, Prasser C, Rochon J, et al. The effects of midazolam on intraocular pressure in children during examination under sedation. Br J Ophthalmol 2011;95:1102-5.

3 Mikhail M, Sabri K, Levin AV. Effect of anesthesia on intraocular pressure measurement in children. Surv Ophthalmol 2017;62:648-58.

4 Chan A-W, Tetzlaff JM, Gøtzsche PC, et al. Spirit 2013 explanation and elaboration: guidance for protocols of clinical trials. $B M J$ 2013;346:e7586.

5 Montazeri K, Dehghan A, Akbari S. Increase in intraocular pressure is less with propofol and remifentanil than isoflurane with remifentanil during cataract surgery: a randomized controlled trial. Adv Biomed Res 2015;4:55.

6 Hanna SF, Ahmad F, Pappas ALS, et al. The effect of propofol/ remifentanil rapid-induction technique without muscle relaxants on intraocular pressure. J Clin Anesth 2010;22:437-42.

7 Termühlen J, Gottschalk A, Eter N, et al. Does general anesthesia have a clinical impact on intraocular pressure in children? Paediatr Anaesth 2016;26:936-41.

8 Barclay K, Wall T, Wareham K, et al. Intra-ocular pressure changes in patients with glaucoma. Comparison between the laryngeal mask airway and tracheal tube. Anaesthesia 1994;49:159-62.

9 Deramoudt V, Gaudon M, Malledant Y, et al. [Effects of propofol on intraocular pressure in surgery of strabismus in children]. Ann Fr Anesth Reanim 1990;9:1-5.

10 Derbyshire DR, Smith G, Achola KJ. Effect of topical lignocaine on the sympathoadrenal responses to tracheal intubation. $\mathrm{Br} \mathrm{J}$ Anaesth 1987;59:300-4.

11 Watcha MF, Chu FC, Stevens JL, et al. Intraocular pressure and hemodynamic changes following tracheal intubation in children. $J$ Clin Anesth 1991;3:310-3.

12 Watcha MF, White PF, Tychsen L, et al. Comparative effects of laryngeal mask airway and endotracheal tube insertion on intraocular pressure in children. Anesth Analg 1992;75:355-60.

13 Patel A, Clark SR, Schiffmiller M, et al. A survey of practice patterns in the use of laryngeal mask by pediatric anesthesiologists. Paediatr Anaesth 2015;25:1127-31.

14 Agrawal G, Agarwal M, Taneja S. A randomized comparative study of intraocular pressure and hemodynamic changes on insertion of proseal laryngeal mask airway and conventional tracheal intubation in pediatric patients. J Anaesthesiol Clin Pharmacol 2012;28:326-9.

15 Schäfer R, Klett J, Auffarth G, et al. Intraocular pressure more reduced during anesthesia with propofol than with sevoflurane: both combined with remifentanil. Acta Anaesthesiol Scand 2002;46:703-6.

16 Dominguez A, Garcia-Miguel FJ, Alsina E, et al. Intraocular pressure measurement in children under general anaesthesia with sevoflurane. Eur J Anaesthesiol 2009;26:801-3.

17 Piepho T, Cavus E, Noppens R, et al. S1 guidelines on airway management : Guideline of the German Society of Anesthesiology and Intensive Care Medicine. Anaesthesist 2015;64:27-40.

18 Lenoir RJ. Venous congestion of the neck; its relation to laryngeal mask cuff pressures. Br J Anaesth 2004;93:476-7.

19 Lopes JE, Wilson RR, Alvim HS, et al. Central corneal thickness in pediatric glaucoma. J Pediatr Ophthalmol Strabismus 2007;44:112-7.

20 Levy J, Lifshitz T, Rosen S, et al. Is the tono-pen accurate for measuring intraocular pressure in young children with congenital glaucoma? J Aapos 2005;9:321-5.

21 Perkins ES. Hand-held applanation tonometer. Br J Ophthalmol 1965;49:591-3.

22 Esmael A, Ismail YM, Elhusseiny AM, et al. Agreement profiles for rebound and applanation tonometry in normal and glaucomatous children. Eur J Ophthalmol 2019;29:379-85.

23 Nakakura S. Icare ${ }^{\circledR}$ rebound tonometers: review of their characteristics and ease of use. Clin Ophthalmol 2018;12:1245-53. 